\title{
Consumer Modelling in Support of Interface Design
}

\author{
Timothy J. Maciag, Dominik Slezak, Daryl H. Hepting \\ University of Regina \\ Department of Computer Science \\ Regina, Saskatchewan, S4S 0A2 \\ \{maciagt, slezak,dhh\}@cs.uregina.ca
}

\begin{abstract}
There is significant interest in developing new methods to design more effective user interfaces for decision support tools in online shopping environments. Many online companies have already begun to provide their consumers with enhanced user interface options, such as the ability to customize and/or personalize their user interface. However, for these enhanced options to produce meaningful, useful results, consumers are often required to input substantial amounts of information, placing a strain on the consumers' cognitive decision-making abilities and disrupting their focus on their immediate decision task(s). In this paper, the authors describe a personalization technique to reduce the amount of consumer information required to develop and deploy systems providing these enhanced options. Over the course of the three experiments, the authors built upon each experiment utilizing a combination of traditional statistical methods and rough set theory. This paper will describe the refined technique and the procedures, algorithms, observations, and analysis of the experiments conducted. As well, a discussion detailing future work will be provided.
\end{abstract}

\section{Introduction}

Millions of consumers engage in e-commerce activities each day, searching, shopping, and purchasing items online. To support the consumer's e-commerce activities many online sites provide consumers with customizable, personalized user interfaces and decision support tools that aid them in finding products matching their individual decisionmaking criteria [7]. Some of these user interfaces and decision support tools are simple, e.g. a basic product search engine enabling categorical search and displaying results in descending order of importance. However, there exist sites, such as Amazon (www.amazon.ca), Ebay (www.ebay.ca), and Chapters (www.chapters.indigo.ca), that provide consumers with more complex decision support tools. These sites enable consumers to customize and personalize their user interface to highlight potential items of interest [17].

For example, Amazon enhances the consumer's shopping experience by providing decision support tools that aid in choosing current and future selections. This is accomplished by keeping a record of the consumer's transaction history and providing consumers the option to compile wish lists, a compilation of items indicated by the consumer as being interesting or desirable. This information is collected and used to formulate product recommendation pages, a customizable and personalized page where consumers can select categories of interest and have recommendations displayed in descending order of importance.

A major drawback to many of these personalization techniques is that they require consumers to compile a considerable profile (preference listing and transaction history) before these enhancements prove meaningful [7]. The authors of this paper, over the course of three experiments, have refined a personalization technique that reduces the amount of information required by consumers. Thus, consumers are provided with a customizable and personalized user interface quickly, enabling them to focus solely on their immediate decision task(s) while gaining a more satisfactory online shopping experience.

The remainder of this paper will be organized as follows: Section 2 will discuss background information and motivation. Section 3 will describe the three experiment designs, algorithms, and procedures. Section 4 will provide a discussion on the results obtained. Finally, Section 5 will provide concluding remarks and a discussion detailing future work.

\section{Background}

Current research in consumer marketing has indicated that consumers generally employ two decision strategies in their decision-making tasks: compensatory and noncompensatory decision strategies [2]. Compensatory decision strategies are used when the decision-maker applies a strict, rationalized thought process based on pre-defined 
preferences, ratings, or rankings to formulate a final decision. The decision-maker will systematically weigh all possible alternatives and options in order to form the best possible decision outcome. Conversely, non-compensatory decision strategies are used when the decision-maker applies a bounded, rationalized thought process in the their decision-making tasks. Bounded rationality, defined by Simon [20], refers to the concept that decision-makers have a limit on their cognitive capacity for reaching fully rationalized decision outcomes. Decision-makers will often arrive at a final decision based on ad hoc, non-compensatory decision strategies based on a variety of factors, including: pre-defined and developing preferences, ratings, rankings, the interface design, and the display of information $[2,8]$.

Research has indicated that consumers often employ different decision strategies given certain contexts and the availability of enhanced functions to support their decisionmaking process $[2,4,8]$. However, since non-compensatory decision-making strategies may not always provide consumers with their most preferred decision outcome(s), as consumers may overlook decision outcomes that may be more suited to their individual preferences, decision support tools should be designed to enable utilization of both decision strategies.

For example, Jedetski et al. [9] describe an experiment to evaluate the consumer satisfaction in online environments wherein consumers were able to either employ compensatory or non-compensatory decision strategies. Their results indicated that decision support tools that enabled compensatory decision strategies provided consumers with a more satisfying online shopping experience. However, Bettman et al. [2] describe that many consumers may not always be willing to employ compensatory decision strategies, and more often than not, consumers apply non-compensatory decision strategies. Thus, the interface should enable consumers to conduct both decision strategies effectively.

Current e-technologies enable designers to develop decision support tools to aid consumers in performing both decision strategies. However, the design of the user interface has a tremendous bearing whether or not consumers will have a satisfying experience $[8,9]$. One design method system designers could use when designing and developing user interfaces to support consumer decision-making tasks is to provide consumers the option to customize and/or personalize their user interfaces. Personalizing the user interface will enhance the consumers cognitive decision-making abilities and aid them in performing their compensatory and non-compensatory decision strategies by highlighting key attributes of interest and featured products matching their individual values.

To provide customization and personalization, designers must initially assess consumer preferences. Throughout the last decade market analysts and consumer researchers have used a number of common techniques to assess and predict consumer preferences, including choice, rating, and ranking [14]. These methods are used in the evaluations described here. The authors of this paper have developed and refined a personalization technique that reduces the amount of consumer information required to develop and deploy customizable, personalized user interfaces.

The next section will describe the personalization technique developed by the authors, the history of development and refinement, and the experiment evaluation procedures used.

\section{Experiment Design}

To support their research, the authors conducted a usability evaluation of a suite of environmental decision support tools for consumer-to-product environmental impact assessment. The rationale behind choosing this type of software application was due to the increasing need to provide more usable systems of this kind in the current marketplace. Such tools have great potential to aid consumers in visualizing the environmental impacts of their everyday product choices. The tools selected for this evaluation were initially developed by the United States Environmental Protection Agency (US-EPA). The US-EPA provided three decision support tools as part of their suite, one of which is illustrated in Figure 1. The authors chose to evaluate the US-EPA tools due to their accessibility and availability online. However, they have since been taken off-line.

The US-EPA tools enabled consumers to make product selections in the context of environmentally friendly cleaning products. The original design of the experiment was to evaluate the US-EPA tools in comparison to a decision support tool developed by the authors of this paper called cogito [6]. The cogito system provides designers the ability to develop software application extensions that utilize cogito's core system functionality. For this experiment, the authors designed two software application extensions to conduct an evaluation with the US-EPA tools, one of which is illustrated in Figure 2.

The cleaning product database consisted of information that was accumulated from the United States General Service Administration (US-GSA). The information provided by the US-GSA was incorporated into each of the US-EPA tools and cogito software application extensions, and enabled consumers to filter 29 cleaning products using eight product attributes. The eight product attributes included:

1. Skin Irritation (skin): Refers to the presence of chemicals in the cleaning product that cause redness or swelling of skin. Attribute values range from the most preferable to least preferable value, i.e. negligi- 


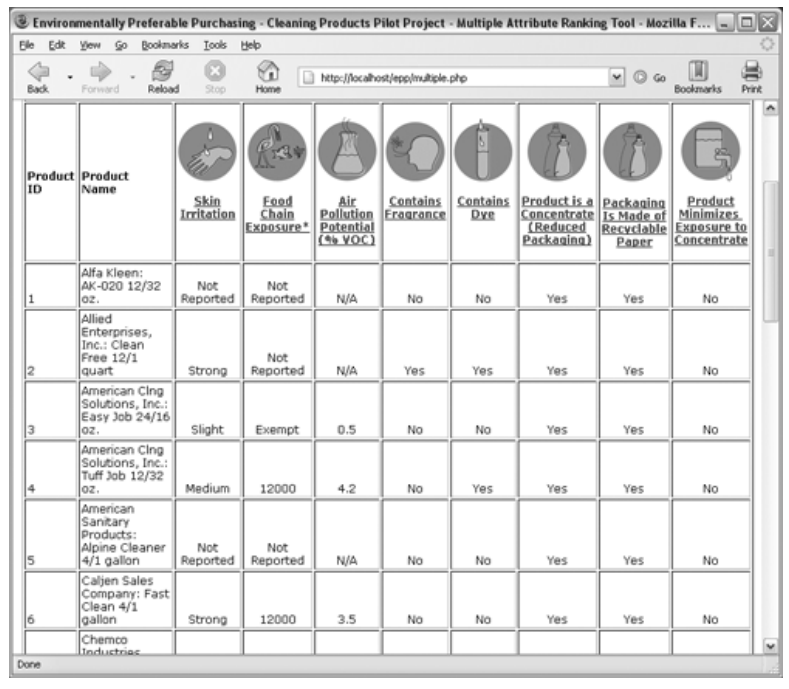

Figure 1. Screen capture of one of the USEPA tools. The US-EPA provided three tools, a single attribute ranking tool (pictured), a multiple attribute ranking tool (providing multiple product filtering options), and a weighted attribute ranking tool (providing filtering by weighting attribute importance).

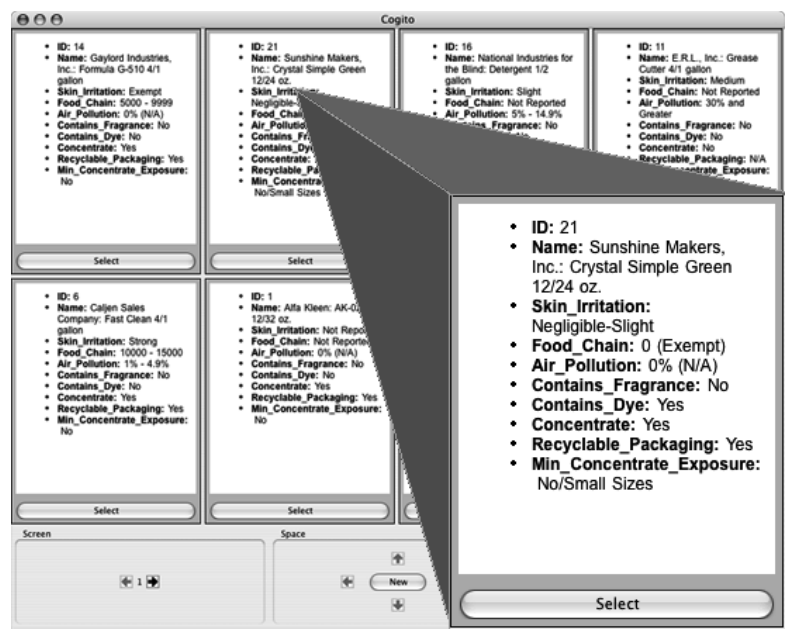

Figure 2. Screen capture of the cogito software application extension derived from the US-EPA tools. The authors designed two software application extensions, a textual interface (pictured) and a graphical interface (using nightingale rose illustrations to represent the cleaning products). A product cell is magnified for display purposes. ble, slight, moderate, or strong. A special skin irritation value of "exempt" signifies that there is less than $5 \%$ (by weight) chemical component in the product.

2. Food Chain Exposure (fce): Refers to ingredients in cleaning products that have the potential to be introduced into the food chain by being consumed by smaller aquatic plants and animals which are than consumed by larger animals. Food chain exposure is measured by calculating a products bioconcentration factor (BCF). Products with a BCF less than 1000 or a BCF of "exempt" are more preferable.

3. Air Pollution Potential (air): Refers to products that may contain volatile organic compounds (VOC), i.e. compounds that have the potential to form atmospheric pollutants, e.g. smog. These pollutants can cause eye, nose, throat, and lung irritation, as well as trigger asthma attacks. The lower the VOC, the more preferable the product with a special value of "N/A" (not applicable), which indicates that there are no VOCs of concern present, being the most preferable.

4. Product Contains Fragrances (frag): Refers to fragrances that are added to the cleaning product to improve, or mask, its "natural" odor.

5. Product Contains Dye (dye): Refers to dyes that have been added to the cleaning product to change the colour of the product.

6. Product uses Recyclable Packaging (rec): Refers to cleaning products that are packaged using recyclable packaging.

7. Product is a Concentrate (con): Refers to cleaning products that are packaged using reduced packaging, e.g. packaging the cleaning product in a recyclable plastic bag which acts as a refiller for use in its original packaging.

8. Product Reduces Exposure to Concentrate (exp): Refers to cleaning products that reduce exposure to concentrated packaging. Concentrates have potential to increase health risk as it may place the consumer at greater exposure to potentially hazardous ingredients of the product.

The interface design of the US-EPA tools and the $\operatorname{cog}$ ito system both provided consumers with the option to employ either compensatory and/or non-compensatory decision strategies. However, the tools did not enable consumers to customize or personalize their interface. Although the results of the preliminary usability evaluation indicated that users preferred the cogito system in terms of user response times, decision task scores, and satisfaction 
(based on interface preference and user comments) [13], the authors hypothesize that if enhancements such as personalization were included into the original interface designs, user satisfaction levels would increase. However, instead of requiring consumers to build a substantial consumer profile in order for these enhanced user interface options to prove meaningful, the authors wanted to evaluate the possibility of reducing the amount of information required by consumers, while still providing them with meaningful and useful interface designs. Even though the experiment technique was evaluated in the context of environmentally preferable purchasing, the authors hypothesize that that the personalization technique would work universally, regardless of application type.

\subsection{Usability Evaluation}

The information used in the experiments was accumulated from a usability evaluation conducted by the authors. Participants recruited for the evaluation were primarily undergraduate students from the University of Regina (The authors used the University of Regina Computer Science Participant Pool to recruit participants. Participants were eligible to receive a bonus credit in a participating computer science course of their choosing as reward for their participation). It is important to note that the number of participants grew over the course of the three experiments described here. In the first and second experiment, 48 participants were recruited. For the refined experiment described later in this paper, eight additional participants were recruited. One of the tasks that the participants were required to complete included ranking the eight system attributes (described in the preceding section) based on their perceived importance. Each attribute was ranked using the following four point scale: unimportant, somewhat important, important, and very important. The information obtained from the preference elicitation formed the basis for the first two experiments.

\subsection{Rough Sets}

Rough set theory [15] provided the foundation for all three experiments described in this paper. Rough Set theory was developed by Z. Pawlak in the early 1980's and provides techniques for representing uncertainty in information systems [21]. The main concept of rough set theory is the notion of indiscernibility relations, which leads to the reduction of knowledge [16]. Information is displayed in decision tables, consisting of objects (represented in the rows) and attributes and decision classes (represented in the columns). Knowledge reduction techniques can be applied to find redundant attributes in the decision table. Once the redundant attributes are discarded, the remaining indispens- able attributes form what are referred to as reducts, i.e. those features within the decision table that are necessary to discern the objects therein.

\subsection{Summary of Preliminary Experiments}

Two preliminary experiments testing the authors' personalization technique have previously been completed, the second experiment building on results obtained from the first.

The first experiment, conducted by Maciag and Hepting [11], was designed to evaluate the quality of each of the eight system attributes as decision variables. The participants' attribute rankings were discretized into those attributes participants ranked as important or very important and all others. A train and test procedure was conducted; splitting the 48 participants and their associated rankings into randomly constructed training and testing sets. A decision table was constructed where each of the eight attributes were systematically evaluated as a decision variable. Rough set reduction methods were performed using the Rough Set Exploration System (RSES) [1].

Results indicated that when two of the eight attributes were assigned as decision variables (product contains dye and product reduces exposure to concentrate), up to $50 \%$ of the remaining attributes could be discarded while still providing a high classification ( $80 \%$ or higher) of those participants in the testing set. For example, when the attribute product contains dye was assigned as the decision variable, one reduct containing three attributes: skin irritation, food chain exposure, and product contains fragrance was formed. When testing the classification of those participants in the testing set, $91 \%$ of the participants therein were successfully classified. Although these results were encouraging, on further analysis, the two attributes that provided high classification accuracy were largely biased in terms of how the participants ranked them. Thus, the authors questioned whether using this method would provide consumers with personalized interfaces that produced long-lasting useful and meaningful results. For this reason, the authors decided to refine their personalization technique.

The second experiment, conducted by Maciag et al. [12], refined the technique in the first experiment by incorporating a combination of traditional clustering techniques and rough set theory. Similarly as in the first experiment, the participants' attribute rankings were discretized according to those system attributes ranked as important or very important and all others. Different to the first experiment; participants were initially clustered into groups bearing similar attribute preferences (using the k-means clustering algorithm provided by SPSS (www.spss.com), Euclidean distance metric). The resulting participant clusters determined the decision variable (two clusters were formulated thus, 
there were two decision variables). As in the first experiment a training and testing set was randomly constructed and rough set reduction and classification methods were performed using RSES.

Results indicated that of the eight system attributes, only two (product is a concentrate, product uses recyclable packaging) were needed to fully discern participant groups (classification accuracy of $100 \%$ ). Thus, consumers would not have to develop a considerable user profile for these enhanced options to prove meaningful. They would simply need to state, upon system initialization, their preferences pertaining to the two essential attributes required to discern consumer clusters. Although these results were encouraging, and improved on the results obtained from the first experiment, consumer research has shown that traditionally, consumers may change their stated preferences at the time of transaction, selecting products that may only match some of their original preferences [10, 19]. Thus, the authors questioned whether only using attribute preferences as the basis for evaluation was valid and further refined their technique to account for this issue.

The following section will describe the revision to the authors' personalization technique and will provide results obtained from a third experiment conducted.

\subsection{Revised Experiment Design}

Using the same data obtained from the usability evaluation conducted previously (with some additional participant data; 8 new participants thus, $x=56$ ), the authors sought to answer the following:

- Is there a relationship between stated consumer preferences and their product selections?

- Using this information can we reduce the amount of information required by consumers to develop and deploy meaningful, personalized user interfaces?

As part of the usability evaluation described previously, each participant was also asked to select which of the cleaning products provided by the tools they would consider for their own personal use. This information was used as the basis for this refined experiment. Alternatively to clustering the participants into groups with similar attribute preferences, the 29 cleaning products in the database were clustered into groups of similar products. The authors conducted a survey of four distance metrics commonly used in clustering procedures, i.e. Pearson, Spearman, Euclidean, and maximum, in support of finding the best possible cluster results (as the choice of distance metric will have a significant impact on the validity of the resulting clusters). The $R$ project for statistical computing (http://www.r-project.org/) was used to perform the clustering procedure. Results are illustrated in Figures 3, 4, 5, and 6.

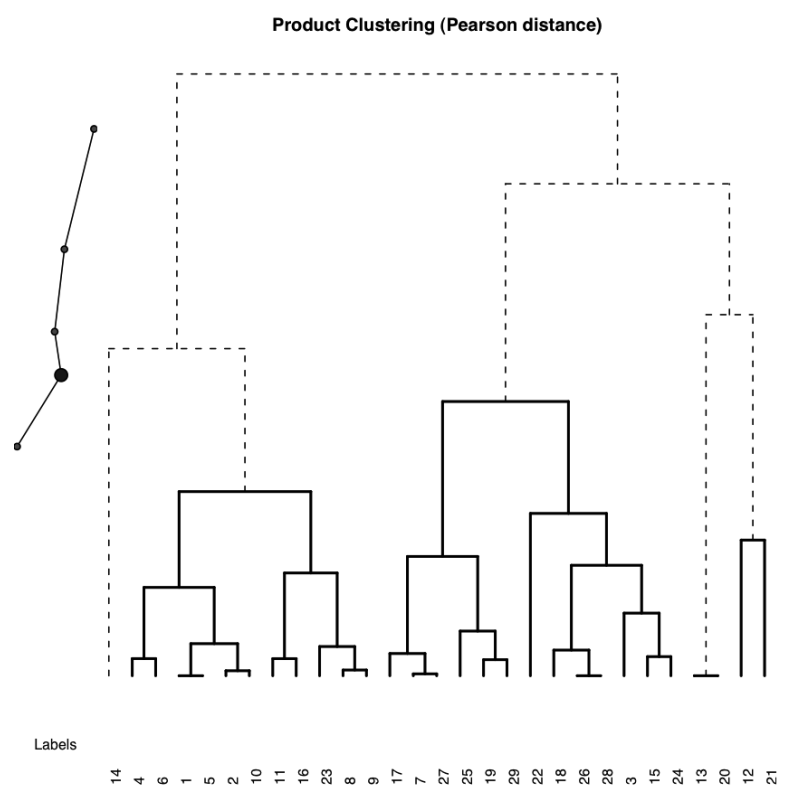

Figure 3. Product clustering using Pearson distances. Five product clusters were formulated. Illustrated on the left-hand side is the result from Hubert's $\Gamma$ statistic.

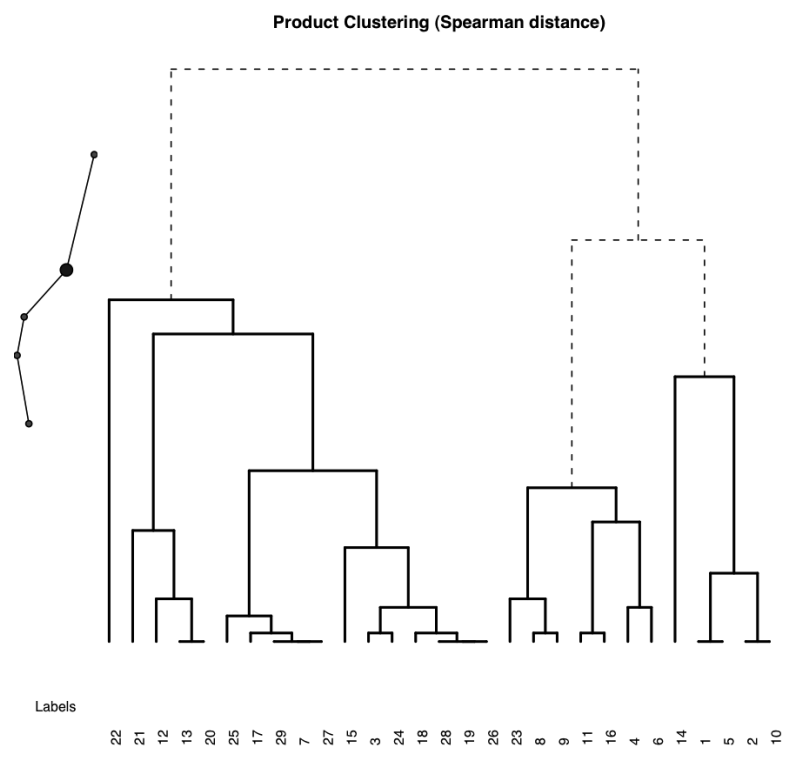

Figure 4. Product clustering using Spearman distances. Three product clusters were formulated. Illustrated on the left-hand side is the result from Hubert's $\Gamma$ statistic. 


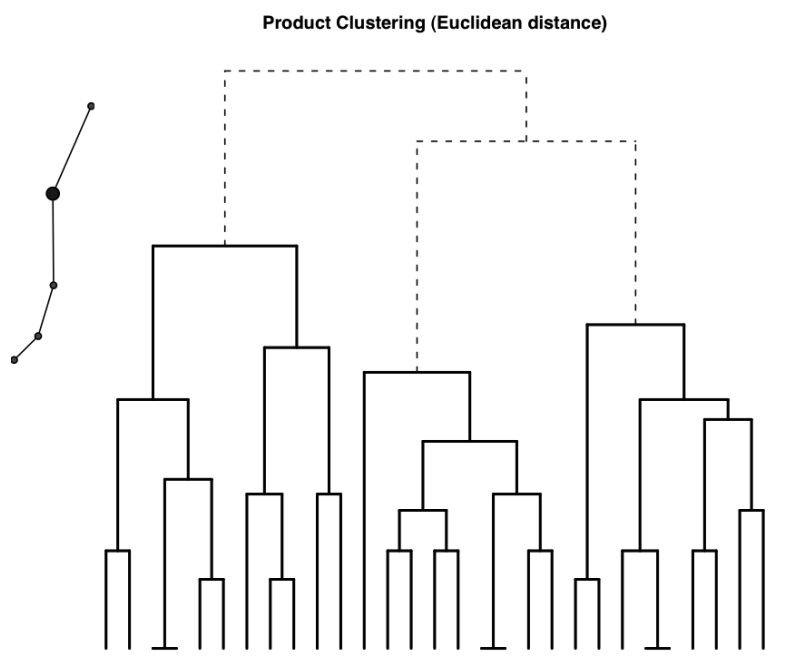

Labols

$+\operatorname{*nกำก~}$

Figure 5. Product clustering using Euclidean distances. Three product clusters were formulated. Illustrated on the left-hand side is the result from Hubert's $\Gamma$ statistic.

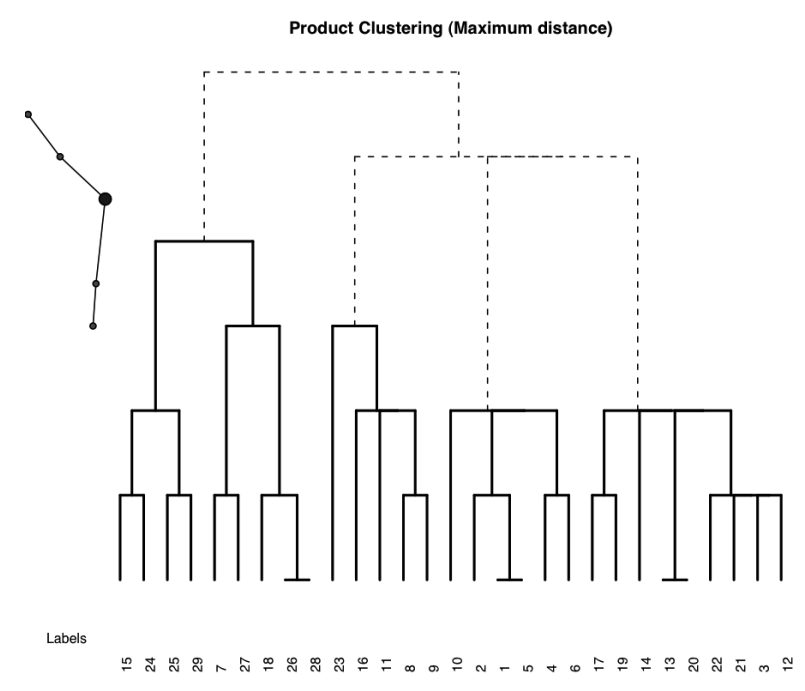

Figure 6. Product clustering using maximum distances. Four distinct product clusters were formulated. Illustrated on the left-hand side is the result from Hubert's $\Gamma$ statistic.
To assist in validating the number of clusters for each distance metric surveyed, Huberts $\Gamma$ statistic $[3,5]$ was used. Hubert's $\Gamma$ statistic assists in assessing the "degree of match" for objects residing within resulting clusters [5]. Illustrated on the left-hand side of Figures 3, 4, 5, and 6 are line plots indicating the results from Hubert's $\Gamma$ statistic. Significant changes in the line plot, such as the visual representation of a knee (commonly referred to as Hubert's knees), are used to indicate the number of clusters that the objects should be grouped in [3]. When observing the four figures, only Figure 6, which used the maximum distance metric, illustrates a distinct Hubert knee. Thus, the authors chose to cluster the 29 products using the maximum distance metric.

Each participant was assigned to the product cluster corresponding to his or her product selection; that value became the participants' new decision variable. For example, if a participant selected product 12 , the participant's decision variable would equal 4 (See Table 1). Some participants responded with multiple selections. These participants were handled by assigning them to the product cluster corresponding to the one that covered the majority of their selections. For example, if a participant responded with five selections, three of which belonged to the same cluster, the participant was assigned to that product cluster accordingly. If the participant's selections were evenly distributed among product clusters, e.g. if the participant responded with two products, each represented in different product clusters, they were randomly assigned to one of the product clusters accordingly. Some participants did not provide a product selection and these participants were omitted from further observation.

Table 1. Product Cluster Results (4 product clusters, maximum distance metric)

\begin{tabular}{|c|l|}
\hline Cluster & Products \\
\hline \hline 1 & $15,24,25,29,7,27,18,26,28$ \\
\hline 2 & $23,16,11,8,9$ \\
\hline 3 & $10,2,1,5,4,6$ \\
\hline 4 & $17,19,14,13,20,22,21,3,12$ \\
\hline
\end{tabular}

Based on the product selections provided by the participants' responses, only three of the four product clusters included products selected by the participants (none of the participants selected products belonging to the first cluster, see Table 1). A decision table was constructed using the participants' non-discretized attribute rankings (data from the four point ranking scale as described in Section 3) and their discretized attribute rankings (as used in the first two experiments) as the decision table objects and corresponding 
attributes (non-discretized + discretized $=16$ decision table attributes). Similarly as in the previous experiments, training and testing sets were randomly constructed and rough set reduction and classification methods were performed using RSES.

\section{Results}

The genetic algorithm method provided by RSES was used to form the ten best reducts. The criteria used to find the best reducts included those reducts that contained the fewest number of attributes while maintaining a high positive region (The positive region, or lower approximation, encapsulates all objects that belong without question to a specific classification based on the reduction procedure [11]). The resulting reducts are shown in Table 2.

Table 2. Formulated Reducts (Top ten). Refer to Section 3 for attribute abbreviation definitions. (Attributes additionally labelled with " $d$ " refer to the discretized rankings)

\begin{tabular}{|c|c|c|l|}
\hline Num & Size & Pos. Region & Reducts \\
\hline \hline 1 & 2 & 1 & dye, con \\
\hline 2 & 2 & 1 & fce, rec \\
\hline 3 & 2 & 1 & fce, con \\
\hline 4 & 2 & 1 & skin, exp \\
\hline 5 & 2 & 1 & fce, $\exp$ \\
\hline 6 & 2 & 1 & air, con \\
\hline 7 & 3 & 1 & frag, dye, $\exp$ \\
\hline 8 & 2 & 1 & rec, fce $d$ \\
\hline 9 & 2 & 1 & con, fce $d$ \\
\hline 10 & 2 & 1 & con, dye $e_{d}$ \\
\hline
\end{tabular}

The reducts formulated from the data in the training set were used to evaluate whether or not participants in the testing set could be correctly classified. Results from the evaluation were quite encouraging. Results indicated the majority of reducts contained only two of the 16 decision table attributes. Thus, generally only two attributes were found to be necessary to satisfactorily discern the participants in the testing set. The overall classification accuracy was found to be $87 \%$, with a total coverage $88 \%$. To further the analysis, the authors conducted an in-depth evaluation to determine which of the ten reducts provided the best classification accuracy and total coverage. Results are described in Table 3.

The results obtained from the third experiment provide indication that there does exist some relationship between product selections and attribute preferences and that the possibility does exist to reduce the amount of consumer input required to develop and deploy customizable and per-
Table 3. In-depth Reduct Analysis (top three results are emphasized). Refer to Section 3 for attribute abbreviation definitions. (Attributes additionally labelled with " $d$ " refer to the discretized rankings)

\begin{tabular}{|c|c|c|c|}
\hline Num & Reducts & $\begin{array}{c}\text { Classification } \\
\text { Accuracy }\end{array}$ & $\begin{array}{c}\text { Total } \\
\text { Coverage }\end{array}$ \\
\hline 1 & dye, con & $100 \%$ & $77 \%$ \\
\hline 2 & fce, rec & $87 \%$ & $88 \%$ \\
\hline 3 & fce, con & $100 \%$ & $84 \%$ \\
\hline 4 & skin, exp & $100 \%$ & $88 \%$ \\
\hline 5 & fce, exp & $100 \%$ & $82 \%$ \\
\hline 6 & air, con & $100 \%$ & $82 \%$ \\
\hline 7 & frag, dye, exp & $100 \%$ & $59 \%$ \\
\hline 8 & $r e c, f c e_{d}$ & $87 \%$ & $88 \%$ \\
\hline 9 & con, fce $e_{d}$ & $100 \%$ & $\mathbf{8 8 \%}$ \\
\hline 10 & con, dye $e_{d}$ & $100 \%$ & $\mathbf{8 8 \%}$ \\
\hline
\end{tabular}

sonalized user interfaces quickly and with ease. Based on the results described in Table 3, three of the ten reducts (in bold text) provide highly successful classification accuracy and coverage. Thus, a decision support tool could be designed to randomly choose one of these three reducts to obtain consumer information. Since consumers would only be required to state their preferences according to those attributes belonging to one of the randomly chosen reducts in Table 3, the consumers will gain a customizable, personalized user interface quickly, highlighting those attributes that the consumer values and products that they may consider purchasing. Thus, the consumers will be able to focus solely on their immediate decision task(s), increasing their chances of formulating satisfactory decision outcome(s).

\section{Conclusion}

This paper described a refined technique for personalizing user interfaces using as little consumer information as possible in support of enhancing and providing more adequate support for consumer decision strategies. Since many consumers often employ both compensatory and non-compensatory decision strategies with respect to their decision-making tasks in online shopping environments, the authors hypothesize that enhancing the user interface by providing customizable, personalized user interfaces will significantly increase consumer productivity and satisfaction levels when utilizing these types of decision support tools.

The authors conducted a usability evaluation, forming the basis for the three experiments described. The first 
two experiments considered only consumer attribute preferences as the basis for developing a personalization technique. However, current research in the field of consumer marketing has indicated that many consumers often define their preferences at the time of purchase, thus often employing a non-compensatory decision strategy where preferences may change. The third experiment was designed to consider both, attribute preferences and previously selected products as the basis for refinement. Although the authors acknowledge that many e-commerce sites already use this approach (preferences and transaction history) and provide consumers with these enhanced options, the personalization technique that the authors describe in this paper significantly reduced the amount of information required by consumers (only two of the 16 attributes were needed to discern the different consumer types). Consumers will only be required to state their values pertaining to the attributes as represented by one of the reducts as opposed to developing a large consumer profile before these enhanced options produce meaningful designs and useful results.

The results that were obtained from the third experiment were quite encouraging. Future work will include testing the refined personalization technique with real consumers to test the validity of the technique. This will include conducting paper and pencil and scenario-based prototype user evaluations [18]. Consumer satisfaction levels and decision accuracy will be evaluated and analyzed accordingly.

\section{References}

[1] J. Bazan and M. Szczuka. The rough set exploration system. Transactions on Rough Sets 3, pages 37-56, 2005.

[2] J. R. Bettman, M. F. Luce, and J. W. Payne. Constructive consumer choice processes. Journal of Consumer Research, 25(3):187-217, 1998.

[3] J. C. Bezdek and N. R. Pal. Some new indexes of cluster validity. IEEE Transactions on Systems, Man, and Cybernetics, 28(3):301-315, 1998.

[4] R. Dhar, S. M. Nowlis, and S. J. Sherman. Comparison effects on preference construction. Journal of Consumer Research, 26(3):293-306, 1999.

[5] M. Halkidi, Y. Batistakis, and M. Vazirgiannis. Cluster validity methods: Part 1. Special Interest Group on Management of Data (SIGMOD) Record, 31(2):40-45, 2002.

[6] D. H. Hepting. Towards a visual interface for information visualization. In Proc. Information Visualization (IV), pages 295-302, 2002.

[7] S. Holland, M. Ester, and W. Kiessling. Preference mining: A novel approach on mining user preferences for personalized applications. In Proc. Principles and Practice of Knowledge Discovery in Databases (PKDD), 2003.

[8] C. K. Hsee and F. Leclerc. Will products look more attractive when presented separately or together? Journal of Consumer Research, 25(2):175-186, 1998.
[9] J. Jedetski, L. Adelman, and C. Yeo. How web site decision technology affects consumers. IEEE Internet Computing, pages 72-79, 2002.

[10] M. F. Luce, J. W. Payne, and J. R. Bettman. Emotional tradeoff difficulty and choice. Journal of Marketing Research, 36(2):143-159, 1999.

[11] T. Maciag and D. H. Hepting. Analysis of user classifiers for personalization of environmental decision support system interfaces. In Proc. Artificial Neural Networks in Engineering (ANNIE), 2005.

[12] T. Maciag, D. H. Hepting, and D. Slezak. Personalizing user interfaces for environmental decision support systems. In Proc. Rough Sets and Soft Computing in Intelligent Agent and Web Technology, 2005.

[13] T. J. Maciag. An evaluation of user interfaces for environmental decision support systems. Master's thesis, University of Regina, 2005.

[14] S. M. Nowlis and I. Simonson. Attribute-task compatibility as a determinant of consumer preference reversals. Journal of Marketing Research, 34(2):205-218, 1997.

[15] Z. Pawlak. Rough Sets, Theoretical Aspects of Reasoning About Data. Kluwer Academic Publishers, 1991.

[16] Z. Pawlak, J. Grzymala-Busse, R. Slowinski, and W. Ziarko. Rough sets. Communications of the ACM, 38(11):89-95, 1995.

[17] G. Rossi, D. Schwabe, and R. Guimaraes. Designing personalized web applications. In Proc. World Wide Web $(W W W)$, pages 275-284, 2001.

[18] M. B. Rosson and J. M. Carroll. Usability Engineering, Scenario-based Development of Human-Computer Interaction. Morgan Kaufmann Publishers, 2002.

[19] B. Shiv and J. Huber. The impact of anticipating satisfaction on consumer choice. The Journal of Consumer Research, 27(2):202-216, 2000.

[20] H. A. Simon. A behavioral model of rational choice. Economics, 69:99-118, 1955.

[21] R. R. Tan. Rule-based life cycle impact assessment using modified rough set induction methodology. Environmental Modelling and Software (EMS), 20, 2005. 